\title{
Collective Choice Rules on Convex Restricted Domains
}

Citation for published version (APA):

Storcken, A. J. A. (2008). Collective Choice Rules on Convex Restricted Domains. Maastricht University School of Business and Economics. METEOR Research Memorandum No. 003 https://doi.org/10.26481/umamet.2008003

Document status and date:

Published: 01/01/2008

DOI:

10.26481/umamet.2008003

Document Version:

Publisher's PDF, also known as Version of record

\section{Please check the document version of this publication:}

- A submitted manuscript is the version of the article upon submission and before peer-review. There can be important differences between the submitted version and the official published version of record.

People interested in the research are advised to contact the author for the final version of the publication, or visit the DOI to the publisher's website.

- The final author version and the galley proof are versions of the publication after peer review.

- The final published version features the final layout of the paper including the volume, issue and page numbers.

Link to publication

\footnotetext{
General rights rights.

- You may freely distribute the URL identifying the publication in the public portal. please follow below link for the End User Agreement:

www.umlib.nl/taverne-license

Take down policy

If you believe that this document breaches copyright please contact us at:

repository@maastrichtuniversity.nl

providing details and we will investigate your claim.
}

Copyright and moral rights for the publications made accessible in the public portal are retained by the authors and/or other copyright owners and it is a condition of accessing publications that users recognise and abide by the legal requirements associated with these

- Users may download and print one copy of any publication from the public portal for the purpose of private study or research.

- You may not further distribute the material or use it for any profit-making activity or commercial gain

If the publication is distributed under the terms of Article $25 \mathrm{fa}$ of the Dutch Copyright Act, indicated by the "Taverne" license above, 


\section{Ton Storcken}

Collective Choice Rules on Convex Restricted Domains

$\mathrm{RM} / 08 / 003$

JEL code: D70

\section{METE@R}

Maastricht research school of Economics of TEchnology and ORganizations

Universiteit Maastricht

Faculty of Economics and Business Administration P.O. Box 616

NL - 6200 MD Maastricht

phone : ++31433883830

fax : ++31433884873 


\title{
Collective Choice Rules on Convex Restricted Domains
}

\author{
Ton Storcken \\ Department of Quantitative Economics \\ Maastricht University
}

February 2008

\begin{abstract}
We study sets of preferences that are convex with respect to the betweeness relation induced by the Kemeny distance for preferences. It appears that these sets consist of all preferences containing a certain partial ordering and the other way around all preferences containing a given partial ordering form a convex set. Next we consider restricted domains where each agent has a convex set of preferences. Necessary and sufficient conditions are formulated under which a restricted domain admits unanimous, strategy-proof and non-dictatorial choice rules. Loosly speeking it boils down to admitting monotone and non-image-dictatorial decision rules on two alternatives where the other alternatives are completely disregarded.
\end{abstract}

\section{Introduction}

One attempt to avoid the well-known impossibility theorems of Arrow and Gibbard-Satterthwaite on collective decision making is dropping the unrestricted domain condition. It means that the individuals taking part in this collective decision potentially may have any preference of a certain kind, e.g. linear orderings or weak orderings over the collective alternatives on which has to be decided. Relaxing this condition leads to so-called restricted domains. Here agents potentially may only have preferences from a subset of this set of preferences or it leads to domains for collective decision making in which all preferences are potentially possible but certain combinations of these are only allowed. Here like in e.g. Kalai e.a.[1977] and Kalai e.a.[1980] and Ritz[1985] we investigate domains allowing 'reasonable' collective decision rules, where at these domains the individual preferences may be chosen independently. Hence, every individual is characterized by a set of admissible preferences being a subset of all possible preferences. Moreover, we assume that any preference which is an intermediate of two admissible preferences is admissible as well. 
There are many studies on domain restrictions (See for a general overview e.g. Gaertner [2002]). Examples on restrictions of combinations of individual preferences can for instance be found in the well-known literature on characterizing domains which allow for consistent pairwise majority decisions. (See e.g. Sen e.a.[1969] and Inada [1964]). A well-known example on restrictions which allow for independent individual preferences is that of single peaked preferences. (See e.g. Arrow[1978], Black[1948] and Moulin[1980] being just an arbitrary choice of this strand of literature).

Having two preferences by repeatedly swapping consecutive ordered pairs of alternatives the one preference can be obtained from the other. The domain restriction imposed here demands that all the intermediate results in this swapping process are admissible if of course the starting two preferences are admissible. In this way we guarantee a natural assumption that the set of admissible preferences of an individual allows for a continuous change of these preferences. For instance let at one admissible preference alternative $a$ be strictly preferred to $b$ and $b$ strictly to $c$ (assuming transitivity we also have that $a$ is strictly preferred to $c$ ) and at an other preference let $c$ be strictly preferred to $a$ and $a$ strictly to $b$. So, in both preferences $a$ is strictly preferred to $b$, where in the one $c$ is worst among these three and in the other $c$ is best. As both these preferences are admissible it is natural to assume that also the intermediate of these two where $c$ is ordered between $a$ and $b$ is admissible. Precisely this is captured by the requirement that the intermediate of two admissible preferences is admissible.

Consider the Kemeny distance between preferences. For any two preferences it counts the pairs that are differently ordered in these two preferences (see also Kemeny e.a. [1962]). It is easy to see that a preference is intermediate of two given preferences if the Kemeny distance between these two equals the sum of the Kemeny distances from each of these to the intermediate. That is the triangle inequality holds with equality. Geometrically speaking this intermediate is on the segment between the other two. Hence, the condition on the intermediate is just a convexity requirement with respect to the Kemeny distance. That is we demand that the set of admissible preferences is a convex subset of the set of all preferences. Clearly such a convex set may range from the set of all preferences, the least restrictive situation as there is no restriction imposed, to a singleton set the most restrictive case. It appears that a convex subset of the set of linear orderings, i.e. the set of all complete, antisymmetric and transitive relations, can be characterized as follows. For every convex set there is a partial ordering, i.e. reflexive, antisymmetric and transitive relation, which is contained in all preferences belonging to this set and moreover any linear ordering containing this partial ordering belongs to this convex set. So, a subset of the set of linear orderings is convex if, and only if, there is a partial ordering such that this convex set equals the set of all linear orderings that contain this partial ordering.

Next consider the case of restricted domains where the sets of individually admissible preferences are convex subsets of the set of linear orderings. The set of admissible preferences of a specific individual is characterized by a partial ordering that is contained in all its admissible preferences. Therefore this partial ordering can be seen as a part of the preference of that individual 
which is known in advance. It is called a priori information. Hence, the restricted domain at hand is completely determined by these individual partial orderings. A natural question is which combination of individual partial orderings determines a restricted domain allowing for non-dictatorial, unanimous and strategy-proof choice rules. We will answer this question with respect to the union of all these partial orderings: the united a priori information. In Storcken[1985] a similar question is studied with respect to Arrow-like welfare functions. These are Pareto-optimal, non-dictatorial welfare functions which are independent of irrelevant alternatives. Roughly speaking such a restricted domain admits Arrow-like welfare functions if not all free triples with respect to the united a priori information are connected. Here a free triple with respect to this a priori information is a triple of alternatives which has no ordered pair of different alternatives in common with this united a priori information. This means that all agents may order these three alternatives in all six possible ways. In the paper at hand the characterization looks at least more restrictive. Loosely speaking these domains allow for unanimous, non-dictatorial and strategy-proof choice rules if there are (at most) two undominated alternatives with respect to the united a priori information. Moreover, the choice rule now is just a monotonic choice rule between these two alternatives.

This difference in conditions on the united a priori information between the welfare function case and the choice function case stems primarily from the nondictatorship requirement. Contrary to a choice function at a welfare function an individual might not be a dictator even if his best alternative is always ordered best in the social ranking as long as somewhere the rest of his preferences is not always copied as the social ranking. Moreover, as the domain in this setting is possibly restricted, not all alternatives might be ranked best by this individual. To be more explicit let $i$ be an individual and $a$ an alternative. Let further the set of admissible preferences of individual $i$ be equal to the set of linear orderings in which $a$ is ordered best. For other individuals let there be no restriction. The hierarchical welfare function in which at any preference profile $a$ is ordered best and all the other alternatives are ordered according to the preference of some fixed individual $j$ different from $i$ is Pareto-optimal, non-dictatorial and independent if irrelevant alternatives. Lemma 3 however shows that this domain does not allow for unanimous, non-dictatorial and strategy-proof choice rules. This shows that although the earlier paper of Storcken[1985] and the present work are based on strongly related idea's, they seem logically independent.

Indeed Lemma's 1 and 2 show that in case there are at least three undominated alternatives with respect to the united a prior information then only dictatorial choice rules are unanimous and strategy-proof. Having this result it is straight forward to see that essentially the case of two undominated alternatives with respect to the united a priori information yield the possibility of non-dictatorial, unanimous and strategy-proof choice rules. Rules that are unanimous and monotonic choices between two alternatives. The cases of one and no undominated alternatives are reduced to the two undominated alternatives case. This however may fail as the example above on one undominated alternative $a$ demonstrates. Lemma's 3 and 4 are on necessary and sufficient 
conditions in order to transform these cases to the case of two undominated alternatives.

The paper is organized as follows. Besides some basic concepts on preferences section 2 is on the character of convex subsets of the set of linear orderings. In section 3 the decision model on convex restricted domains is introduced. Section 4 discusses sufficient and necessary conditions such that these domains admit non-dictatorial, unanimous and strategy-proof choice rules. Section 5 concludes.

\section{Convex sets of preferences}

Let $A$ denote a non-empty and finite set of alternatives and $R$ a relation on $A$. For a non-empty subset $B$ of $A$ the restriction of $R$ to $B$ is denoted by $\left.R\right|_{B}$. The best alternatives with respect to $R$ are defined by best $(R)=\{x \in A \mid(x, y) \in R$ for all $y \in A\}$. The undominated alternatives with respect to $R$ are defined by undom $(R)=\{x \in A \mid(y, x) \notin R$ for all $y \in A\}$. Let $\mathbb{L}(A)$ denote the set of all linear orderings on $A$, i.e. all transitive, complete and anti-symmetric relations on $A$. Cardinality of a set $S$ is denoted by $\# S$.

Let $R^{1}, R^{2}$ and $R^{3}$ be three linear orderings on $A$. Let $B$ be a non-trivial subset of $A$. The relation $\left.\left.R^{1}\right|_{B} \gg R^{2}\right|_{A-B}=\left.\left.R^{1}\right|_{B} \cup R^{2}\right|_{A-B} \cup(B \times(A-B))$ is a relation in which the alternatives in $B$ are ordered as in $R^{1}$ the alternatives in $A-B$ are ordered as in $R^{2}$ and in which all alternatives in $B$ are strictly preferred to all alternatives in $A-B$. As a convention we write $x$ instead of $\left.R^{1}\right|_{\{x\}}$ for alternatives $x$. Further, instead of $(x, y) \in R^{1}$ and $(y, x) \notin R^{1}$ we also write $R^{1}=\ldots x \gg \ldots y \ldots$ The notation $R^{1}=\ldots x \gg y \ldots$ means that $(x, y) \in R^{1}$ and $(y, x) \notin R^{1}$ and in addition there are no alternatives $z$ which are ordered in between $x$ and $y$. The Kemeny distance ${ }^{1}$ between $R^{1}$ and $R^{2}$ is defined by

$$
\delta\left(R^{1}, R^{2}\right):=\frac{1}{2} \#\left[\left(R^{1}-R^{2}\right) \cup\left(R^{2}-R^{1}\right)\right] .
$$

This distance equals the number of setups needed to change relation $R^{1}$ into $R^{2}$. We will call a single setup an elementary change, i.e. $R^{1}$ and $R^{2}$ form such an elementary change if there are two alternatives $a$ and $b$ such that $R^{1}=\left(R^{2}\right.$ $\cup\{(a, b)\})-\{(b, a)\}$. Linear ordering $R^{3}$ is said to be between $R^{1}$ and $R^{2}$ if it is on a shortest path from $R^{1}$ to $R^{3}$, i.e. $\delta\left(R^{1}, R^{2}\right)=\delta\left(R^{1}, R^{3}\right)+\delta\left(R^{3}, R^{2}\right)$. In that case $R^{3}$ can be seen as an intermediate in the change of $R^{1}$ into $R^{2}$ by reversing pairs of alternatives. Clearly this equality holds if, and only if, $\left(R^{1} \cap R^{2}\right) \subseteq R^{3} \subseteq\left(R^{1} \cup R^{2}\right)$. A set of linear orderings say $V$ is said to be convex if for all $R^{1}, R^{2}$ in $V$ and all $R^{3}$ in $\mathbb{L}(A)$ if $R^{3}$ is between $R^{1}$ and $R^{2}$, then $R^{3}$ is in $V$. The following proposition characterizes convexity of sets of preferences.

Proposition 1 Let $V$ be a set of linear orderings. Then $V$ is a convex set if, and only if, there is a partial ordering say $P$ on $A$, i.e. transitive, reflexive and

\footnotetext{
${ }^{1}$ We take here half the Kemeny distance because between complete anti-symmetric relations this distance is an even number.
} 
anti-symmetric, such that

$$
V=\{R \in \mathbb{L}(A) \mid P \subseteq R\} .
$$

Proof. (if-part) Let $V=\{R \in \mathbb{L}(A) \mid P \subseteq R\}$. Furthermore let $R^{1}, R^{2} \in V, R^{3}$ in $\mathbb{L}(A)$ such that $R^{3}$ is between $R^{1}$ and $R^{2}$. It is sufficient to proof that $R^{3}$ is in $V$. Because $R^{3}$ is between $R^{1}$ and $R^{2}$ we have $\left(R^{1} \cap R^{2}\right) \subseteq R^{3}$. As $R^{1}, R^{2} \in$ $V=\{R \in \mathbb{L}(A) \mid P \subseteq R\}$ it follows that $P \subseteq\left(R^{1} \cap R^{2}\right)$. So, $P \subseteq\left(R^{1} \cap R^{2}\right) \subseteq R^{3}$. So, $R^{3} \in\{R \in \mathbb{L}(A) \mid P \subseteq R\}=V$.

(only-if-part) Let $V$ be a convex set of linear orderings. Define $P=\cap\{R \mid R \in$ $V\}$. As all relations in $V$ are reflexive, transitive and anti-symmetric $P$ has these three properties. Now by definition of $P$ it follows that $V \subseteq\{R \in \mathbb{L}(A) \mid P \subseteq R\}$. We have to proof that $\{R \in \mathbb{L}(A) \mid P \subseteq R\} \subseteq V$. To the contrary suppose this is not the case. Then we may find an elementary change, say $R^{1}$ and $R^{2}$ such that $R^{1} \in V$ and $R^{2} \in\{R \in \mathbb{L}(A) \mid P \subseteq R\}-V$. So, there are two alternatives say $a$ and $b$ such that $R^{1}=\left(R^{2} \cup\{(a, b)\}\right)-\{(b, a)\}$. As $V \subseteq\{R \in \mathbb{L}(A) \mid P \subseteq R\}$ it follows that both $R^{1}$ and $R^{2}$ are in $\{R \in \mathbb{L}(A) \mid P \subseteq R\}$. Hence, neither $(a, b)$ nor $(b, a)$ is in $P$. Now by the definition of $P$ and by the convexity of $V$ we may assume the existence of two preferences say $R^{3}$ and $R^{4}$ in $V$ such that $R^{3}=\left(R^{4}\right.$ $\cup\{(a, b)\})-\{(b, a)\}$, that is these two preferences form an elementary change in this same pair $a$ and $b$. Now $\left(R^{1} \cap R^{4}\right) \subseteq R^{2} \subseteq\left(R^{1} \cup R^{4}\right)$, which means that $R^{2}$ is between $R^{1}$ and $R^{4}$. But then by the convexity of $V$ we derive the contradiction $R^{2} \in V$.

\section{Convex restricted domains}

Consider collective choice rules involving finite sets of alternatives $A$ and agents $N=\{1,2, \ldots n\}$ both containing at least two elements. To an agent $i$ we associate $\mathbb{V}^{i}$ a convex subset of the set of linear orderings $\mathbb{L}(A)$. This set $\mathbb{V}^{i}$ represents the set of admissible preferences of this agent. By Proposition1 there is a partial ordering $P^{i}$ such that $\mathbb{V}^{i}=\mathbb{L}\left(P^{i}, A\right)=\left\{R \in \mathbb{L}(A) \mid P^{i} \subseteq R\right\}$. So, this partial ordering $P^{i}$ may be considered as the a priori information we have about agent $i$ 's preference. Let $\mathbb{V}^{N}$ denote the set of all profiles $p$ of individual admissible preferences. The profile $p$ assigns to every agent $i$ a preference $p(i)$ in $\mathbb{V}^{i}$. A (collective) choice rule is a function $f$ from $\mathbb{V}^{N}$ to $A$. It assigns to every profile of individual admissible preferences $p$ a collective choice $f(p)$.

Hereafter choice rules $f$ are studied with respect to the following five conditions:

Unanimity: $f(p)=a$ for all profiles $p$ and alternatives $a$ such that best $(p(i))=$ $a$ for all agents $i$,

Strategy-proofness: $(f(p), f(q)) \in p(j)$ for all agents $j$ and all $j$-deviations $p$ and $q$, i.e. $p, q \in \mathbb{V}^{N}$ with $\left.p\right|_{N-\{j\}}=\left.q\right|_{N-\{j\}}$,

Maskin Monotonicity: for all profiles $p$ and $q$ such that $(f(p), x) \in p(i)$ implies $(f(p), x) \in q(i)$ for all agents $i$ and all alternatives $x$ we have that $f(q)=f(p)$, 
Strong Positive Association: for profiles $p$ and $q$ and alternatives $a$ if for all agents $i$ in $\left.N p(i)\right|_{A-\{a\}}=\left.q(i)\right|_{A-\{a\}}$ and $(a, b) \in p(i)$ implies $(a, b) \in q(i)$ for all alternatives $b \in A$, then $f(q) \in\{a, f(p)\}$.

Non-image-dictatorship: for all agents $j$ there are profiles $p$ such that $f(p) \neq$ best $\left(\left.p(j)\right|_{f\left(\mathbb{V}^{N}\right)}\right)$, where $f\left(\mathbb{V}^{N}\right)=\left\{f(p) \mid p \in \mathbb{V}^{N}\right\}$ is the range of $f$.

Strategy-proofness, Maskin monotonicity, unanimity and strong positive association are standard in literature. We will not comment on these further. Usually a weaker version of non-dictatorship is used it is defined as follows

for all agents $j$ there are profiles $p$ such that $f(p) \neq \operatorname{best}(p(j))$.

Omitting the restriction to the image of $f$ would yield that many convex restricted domains $\mathbb{V}^{N}$ admit odd unanimous, strategy-proofness and nondictatorial choice rules. For instance if there are agents $i$ and $j$ and alternatives $a$ and $b$ such that $(a, b) \in P^{i}$ and $b \in \operatorname{undom}\left(P^{j}\right)$. In that case define $f$ for an arbitrary profile as follows

$$
f(p)=\operatorname{best}\left(\left.p(j)\right|_{A-\{b\}}\right) .
$$

This choice rule is strategy-proof, unanimous and image-dictatorial with image-dictator $j$, but it is non-dictatorial as $b$ is not chosen if it is $j$ 's best alternative. Strategy-proofness follows by the dictatorial nature of this rule. Note that unanimity does not apply to $b$. So, obviously the choice rule is unanimous. Clearly non-image-dictatorship is a stronger condition than non-dictatorship, because $f(p)=\operatorname{best}(p(j))$ implies best $(p(j)) \in f\left(\mathbb{V}^{N}\right)$ and therefore $f(p)=$ $\operatorname{best}(p(j))$ implies $f(p)=\operatorname{best}\left(\left.p(j)\right|_{f\left(\mathbb{V}^{N}\right)}\right)$. To avoid odd possibilities like discussed above we strengthened non-dictatorship to non-image-dictatorship.

Remark 1 Because of the convexity of the domain of individual preferences and the anti-symmetry condition on these individual preferences it follows that strategy-proofness, Maskin monotonicity and strong positive association are all three equivalent to each other. See also Bochet ea.[2005].

Remark 2 Note that for strategy-proof choice rules $f$ from $\mathbb{V}^{N}$ to $A$ and profiles $p$ and $q$ such that $\left.p\right|_{f\left(\mathbb{V}^{N}\right)}=\left.q\right|_{f\left(\mathbb{V}^{N}\right)}$ we have that $f(p)=f(q)$.

\section{Possibilities under Unanimity}

In this section we investigate convex restricted domains $\mathbb{V}^{N}$ which allow for strategy-proof, unanimous and non-image-dictatorial choice rules. Therefore, call $\mathbb{V}^{N}$ a possibility domain if there exists a unanimous, strategy-proof and non-image-dictatorial choice rule $f$ from $\mathbb{V}^{N}$ to $A$. Let $P^{*}=\cup\left\{P^{i} \mid i \in N\right\}$ the union of all a priori information. Related to $P^{*}$ we will formulate necessary and sufficient conditions for convex restricted domains such that these are possibility domains.

The following example deals with a possibility domain that essentially entails the format of any possibility domain in case there are precisely two undominated alternatives. 
Example 1 Let undom $\left(P^{*}\right)=\{a, b\}$, where $a \neq b$. Now any non-imagedictatorial monotonic choice rule on these two alternatives yields a unanimous, strategy-proof and non-image-dictatorial choice rule on $\mathbb{V}^{N}$. To be more explicit let $W_{a}$ and $W_{b}$ be two sets of subsets of $N$ such that for $x \in\{a, b\}$ and for $S \subseteq T \subseteq N$

1. non of these sets in $W_{x}$ is a singleton,

2. $N \in W_{x}$,

3. if $S \in W_{x}$ and $S \subseteq T \subseteq N$, then $T \in W_{x}$,

4. $S \in W_{a}$ if, and only if, $(N-S) \notin W_{b}$.

Define choice rule $f$ from $\mathbb{V}^{N}$ to $A$ for a profile $p$ as follows

$$
\begin{aligned}
f(p) & =a \text { if }\{i \in N \mid(a, b) \in p(i)\} \in W_{a} \\
& =b \text { if }\{i \in N \mid(b, a) \in p(i)\} \in W_{b} .
\end{aligned}
$$

Now $f$ is well-defined because of condition (4). Strategy-proofness follows because of the monotonicity condition (3) and non-image-dictatorship because of condition (1). As $a$ and $b$ are the only undominated elements of $P^{*}$ unanimity has no bite on the other conditions. Unanimity on $a$ and $b$ now follows from condition (2).

Example 1 shows that if the united a priori information $P^{*}$ has two undominated elements, then the domain allows for strategy-proof, non-image-dictatorial and unanimous choice rules. Let $U=\operatorname{undom}\left(P^{*}\right)$. It appears that the cardinality of $\# U$ of $U$ plays an important role in whether or not a domain at hand allows for non-image-dictatorial, unanimous and strategy-proof choice rules. For agents $i$ let $\widetilde{P}^{i}=P^{i} \cup(U \times(A-U))$ and $\widetilde{\mathbb{V}}^{i}=\mathbb{L}\left(\widetilde{P}^{i}, A\right)$. Because $P^{i} \subseteq \widetilde{P}^{i}$, it follows that $\widetilde{\mathbb{V}}^{i} \subseteq \mathbb{V}^{i}$ for all agents $i$. Note that at profiles in $\widetilde{\mathbb{V}}^{N}$ all agents prefer all alternatives in $U$ to all alternatives which are not in $U$. As the alternatives in $U$ are undominated elements of the union of all a priori information $P^{*}$ it follows that no agent a priori prefers any among these. So, if we would restrict all linear orderings in $\widetilde{\mathbb{V}}^{i}$ to $U$ then we would obtain the set of all linear orderings on $U:\left.\widetilde{\mathbb{V}}^{i}\right|_{U}=\mathbb{L}(U)$.

For the four cases $\# U \geq 3, \# U=2, \# U=1$ and $\# U=0$ we will investigate the possibilities of non-image-dictatorial, unanimous and strategy-proof choice rule $h$ from $\mathbb{V}^{N}$ to $A$. Let $h$ be a unanimous and strategy-proof choice rule from $\mathbb{V}^{N}$ to $A$. For the case $\# U \geq 3$ the following two Lemma's yield that $h$ is image-dictatorial.

Lemma 1 Let $\# U \geqslant 3$. Then there is an agent, say $j$, such that for all profiles $p$ in $\mathbb{V}^{N}$ and all alternatives $x \in U$ if best $(p(j))=x$, then $h(p)=x$.

Proof. Let $\widetilde{h}=\left.h\right|_{\widetilde{\mathbb{V}}^{N}}$. As $\widetilde{h}$ inherits unanimity and strategy-proofness from $h$, it follows that $\widetilde{h}$ is Maskin monotone and unanimous. From this we may 
easily deduce that $\widetilde{h}\left(\widetilde{\mathbb{V}}^{N}\right)=U$. Moreover, note that by Maskin monotonicity it now follows that for profiles $p$ and $q$ in $\widetilde{\mathbb{V}}^{N} \widetilde{h}(p)=\widetilde{h}(q)$ if $\left.p\right|_{U}=\left.q\right|_{U}$. So, $\widetilde{h}$ can be seen as a choice rule on $\left.\widetilde{\mathbb{V}}^{N}\right|_{U}=\mathbb{L}(U)^{N}$. Gibbard-Satterthwaite $[1973,1975]$ yields that $\widetilde{h}$ is dictatorial, with dictator say $j$. Now let $p$ be a profile in $\mathbb{V}^{N}$ with best $(p(j))=x \in U$. It is sufficient to prove $h(p)=x$. Take $y \in U-\{x\}$ and profiles $q$ and $r$ in $\mathbb{V}^{N}$ such that $q(j)=\left.x \gg y \gg p(j)\right|_{A-\{x, y\}}$, $r(j)=\left.\left.x \gg y \gg p(j)\right|_{U-\{x, y\}} \gg p(j)\right|_{A-U}, q(i)=\left.y \gg p(i)\right|_{A-\{y\}}$ and $r(i)=$ $\left.\left.y \gg p(i)\right|_{U-\{y\}} \gg p(i)\right|_{A-U}$ for agents $i$ different from $j$. As $r \in \widetilde{\mathbb{V}}^{N}$ and $\widetilde{h}$ is dictatorial with dictator $j$ it follows that $h(r)=x$. Strategy-proofness and unanimity imply that $h(q) \notin A-\{x, y\}$. So, $h(q) \in\{x, y\}$. As $h(r)=x$ Maskin monotonicity implies that $h(q) \neq y$. So, $h(q)=x$. By Maskin monotonicity this implies $h(p)=x$.

Lemma 1 shows that if there are enough undominated elements with respect to the union of a priori information, then there is an agent which is imagedictatorial on all these undominated elements. The lemma hereafter shows that this decisiveness spreads on all alternatives in the image of choice rule $h$.

Lemma 2 Let $\# U \geqslant 3$. Then there is an agent, say $j$, such that for all profiles $p$ in $\mathbb{V}^{N}$ and all alternatives $x \in h\left(\mathbb{V}^{N}\right)$ if best $\left(\left.p(j)\right|_{h\left(\mathbb{V}^{N}\right)}\right)=x$, then $h(p)=x$. Hence, $h$ is image-dictatorial with image-dictator $j$.

Proof. Let $j$ be as in Lemma 1. Let $x \in h\left(\mathbb{V}^{N}\right)$ and best $\left(\left.p(j)\right|_{h\left(\mathbb{V}^{N}\right)}\right)=x$. As $x \in h\left(\mathbb{V}^{N}\right)$ there is a profile $w \in \mathbb{V}^{N}$ with $h(w)=x$. We will construct a list of profiles starting with $w$ and ending at $p$ and prove for all these profiles that their image is $x$ under $h$. Consider profile $v$ a $j$-deviation of $w$ such that $v(j)=p(j)$ and $v(i)=w(i)$ for $i \neq j$. Because of strategy-proofness and $\operatorname{best}\left(\left.p(j)\right|_{h\left(\mathbb{V}^{N}\right)}\right)=x$ it follows from $h(w)=x$ that $h(v)=x$. Now let $a$ and $b$ be two different alternatives in $U$. Without loss of generality assume that $(b, a) \in p(j)$. Consider profile $u$ defined for all $i$ different from $j$ by $u(i)=$ $\left.b \gg v(i)\right|_{A-\{b\}}$. Let $u(j)$ be obtained from $v(j)$ by shifting $a$ just below $x$ and $b$ just below $a: u(i)=\ldots x \gg a \gg b \ldots$. So, $\left.u(j)\right|_{A-\{a, b\}}=\left.v(j)\right|_{A-\{a, b\}}$ and $u(j)$ ranks $x$ just above $a$ just above $b$. Let $\widetilde{u}$ be the $j$-deviation of $v$ such that $\widetilde{u}(j)=u(j)$. Because of $h(v)=x$, Maskin monotonicity implies that $h(\widetilde{u})=x$. But then $h(u) \neq a$, because otherwise Maskin monotonicity would imply that $h(\widetilde{u})=a$ if $h(u)=a$. To see that $h(u) \neq b$ consider profile $\widehat{u}$ a $j$-deviation of $u$ such that $\widehat{u}(j)=\left.a \gg u(j)\right|_{A-\{a\}}$. By Lemma 1 we have that $h(\widehat{u})=a$. Now $h(u) \neq b$, because otherwise Maskin monotonicity would imply that $h(\widehat{u})=b$ if $h(u)=b$. Finally unanimity and strategy-proofness imply that $h(u) \notin h\left(\mathbb{V}^{N}\right)-\{a, b, x\}$. So, $h(u)=x$. Let profile $r$ be the $j$ deviation of $u$ such that $\left.r(j)\right|_{A-\{b\}}=\left.p(j)\right|_{A-\{b\}}$ and $\left.r(j)\right|_{A-\{a\}}=\left.u(j)\right|_{A-\{a\}}$. Maskin monotonicity implies that $h(r)=x$. Let $q$ be the profile such that $q(j)=r(j)$ and for all agents $i$ different from $j$ let $q(i)=\left.b \gg p(i)\right|_{A-\{b\}}$. Maskin monotonicity and $h(r)=x$ implies $h(q) \neq b$. Strategy-proofness and unanimity imply that $h(q) \notin h\left(\mathbb{V}^{N}\right)-\{b, x\}$. So, $h(q)=x$ and Maskin monotonicity now implies $h(p)=x$. 
For the case $\# U=2$ Example 1 shows that there are non-image-dictororial, unanimous and strategy-proof choice rules. Example 2 below shows a case in which such choice rules exist in case $\# U=1$. Moreover, the sufficiency condition implicitly spelled out by this example appears to be necessary as is shown in Lemma 3 below.

Example 2 let $U=\{a\}$ and let $b \in A-\{a\}$ such that $(a, b) \notin P^{1} \cup P^{2}$. Define choice rule $f$ from $\mathbb{V}^{N}$ to $A$ for a profile $p$ as follows

$$
\begin{aligned}
f(p) & =b \text { if both }(b, a) \in p(1) \text { and }(b, a) \in p(2) \\
& =a \text { in all other cases. }
\end{aligned}
$$

Clearly $f$ is non-image-dictatorial and because $U=\{a\}$ unanimity has no bite on alternatives different from a. Hence, by definition it follows that $f$ is unanimous. Strategy-proofness follows because $f$ is essentially a monotonic choice rule between two alternatives.

Actually in Example 2 we defined a choice rule on two alternatives and two agents where neither the rest of the agents nor the rest of the alternatives can influence the outcome. they act just like dummies. It is clear however that we may define choice rules at which more agents, say $j$, can have an impact on the outcome if for such agents $(a, b) \notin P^{j}$. It is also clear if there is only one agent say agent 1 for which $(a, b) \notin P^{1}$ the choice rule defined in Example 2 is image-dictatorial. The following Lemma 3 shows that if $U=\{a\}$ for some alternative $a$, then the existence of an alternative $b \neq a$ such that for two agents $i$ and $j(a, b) \notin P^{i} \cup P^{j}$ is not only a sufficient but also a necessary condition such that $h$ is non-image-dictatorial.

Lemma 3 Let $U=\{a\}$ for some alternative a in $A$. Then the following two are equivalent

1. There are different agents $i$ and $j$ and an alternative $b \in A-\{a\}$ such that $(a, b) \notin P^{i} \cup P^{j}$;

2. There exist unanimous, strategy-proof and non-image-dictatorial choice rules $f$ from $\mathbb{V}^{N}$ to $A$.

Proof. ((1) implies (2)) This implication follows by Example 2.

((2) implies (1)) To prove the contra position of this implication suppose

$(\alpha)$ For all alternatives $b \in A-\{a\}$ there are agents $i_{b}$ such that $(a, b) \in P^{j}$ for all $j \in N-\left\{i_{b}\right\}$;

( $\beta$ ) Choice rules $f$ from $\mathbb{V}^{N}$ to $A$ is unanimous and strategy-proof.

It is sufficient to proof that $f$ is image-dictatorial. By unanimity and $U=$ $\{a\}$ it follows that $a \in f\left(\mathbb{V}^{N}\right)$. If $f\left(\mathbb{V}^{N}\right)=\{a\}$, then $f$ is constant and every 
agent is image-dictator of $f$. Let $\{a, b\}=f\left(\mathbb{V}^{N}\right)$ for some $b \in A-\{a\}$. In view of Remark 2 assumption $(\alpha)$ and unanimity of $f$ yield that for a profile $r$

$$
\begin{aligned}
f(r) & =b \text { if }(b, a) \in p\left(i_{b}\right) \\
& =a \text { in all other cases. }
\end{aligned}
$$

So, agent $i_{b}$ is image-dictator of $f$. Finally, consider the case that $f\left(\mathbb{V}^{N}\right)$ has at least three elements. Let $b, c \in f\left(\mathbb{V}^{N}\right)$ and $a, b$ and $c$ be all different. We first prove that $i_{b}=i_{c}$. Then we show that this agent is imagedictator. To the contrary assume that $i_{b} \neq i_{c}$. In view of assumption $(\alpha)$ and Maskin monotonicity for all $x \in f\left(\mathbb{V}^{N}\right)-\{a\}$ there are profiles $p_{x}$ such that $f\left(p_{x}\right)=x$, for all $j \in N-\left\{i_{x}\right\}$ best $\left(p_{x}(j)\right)=a$ and $p_{x}\left(i_{x}\right)=\ldots x \gg a \ldots$ Consider profile $q$ defined by $q\left(i_{b}\right)=p_{b}\left(i_{b}\right), q\left(i_{c}\right)=p_{c}\left(i_{c}\right)$ and best $(q(j))=a$ for all $j \in N-\left\{i_{b}, i_{c}\right\}$. By assumption $(\alpha)$ it follows that $(a, b) \in p_{c}\left(i_{c}\right)$ and $(a, c) \in p_{b}\left(i_{b}\right)$. Because $f\left(p_{b}\right)=b$ Maskin monotonicity implies $f(q) \neq a$. Let $r$ be a profile defined by $\left.q\right|_{N-\left\{i_{b}, i_{c}\right\}}=\left.r\right|_{N-\left\{i_{b}, i_{c}\right\}}, r\left(i_{b}\right)=\left.a \gg p_{b}\left(i_{b}\right)\right|_{A-\{a\}}$ and $r\left(i_{c}\right)=\left.a \gg p_{c}\left(i_{c}\right)\right|_{A-\{a\}}$. By unanimity $f(r)=a$, so Maskin monotonicity implies $(f(q), a) \in q\left(i_{b}\right)$ or $(f(q), a) \in q\left(i_{c}\right)$. Because of $f(q) \neq a$, assumption $(\alpha)$ implies either $(a, f(q)) \in q\left(i_{b}\right)$ or $(a, f(q)) \in q\left(i_{c}\right)$. Without loss of generality assume $(f(q), a) \in q\left(i_{b}\right)$ and therewith consequently $(a, f(q)) \in q\left(i_{c}\right)$. Consider profile $v$ defined by $\left.v\right|_{N-\left\{i_{b}\right\}}=\left.q\right|_{N-\left\{i_{b}\right\}}$ and $v\left(i_{b}\right)=\left.a \gg p_{b}\left(i_{b}\right)\right|_{A-\{a\}}$ comparing $q$ and $v$ Strong Positive Association yields that $f(v) \in\{a, f(q)\}$. If $f(v)=f(q)$, then $f$ is manipulable by agent $i_{c}$ at profile $v$ towards profile $r$. If $f(v)=a$, then Maskin monotonicity implies the contradiction $f\left(p_{c}\right)=a$. Hence, $i_{b}=i_{c}$. Since $b$ and $c$ were arbitrary choices we may conclude that there is an agent $i$ such that $i=i_{x}$ for all $x \in f\left(\mathbb{V}^{N}\right)-\{a\}$. In order to prove that $i$ is image-dictator of $f$ let $w$ be a profile in $\mathbb{V}^{N}$ such that $x=\operatorname{best}\left(\left.w(i)\right|_{f\left(\mathbb{V}^{N}\right)}\right)$. It is sufficient to prove that $f(w)=x$. To the contrary let $f(w)=y \neq x$. By assumption $(\alpha)$ we have that $(a, z) \in w(j)$ for all $z \in f\left(\mathbb{V}^{N}\right)-\{a\}$ and all $j \in N-\{i\}$. According to Remark 2 we may assume that $a=\operatorname{best}(w(j))$ for all $j \in N-\{i\}$. Because $f(w)=y$ it follows by unanimity and strategyproofness that $(y, a) \in w(i)$. Consider $i$-deviation $u$ of $w$ such that $u(j)=w(j)$ for $j \in N-\{i\}, u(i)=\ldots x \gg a \ldots$ and $\left.u(i)\right|_{A-\{a\}}=\left.w(i)\right|_{A-\{a\}}$. Strong Positive Association implies that $f(u) \in\{a, y\}$. As $(a, y) \in u(i)$ unanimity and startegyproofness imply that $f(u) \neq y$ in case $a \neq y$. So, $f(u)=a$. But then Maskin monotonicity implies $f\left(\bar{p}_{x}\right)=a$, where $\bar{p}_{x}(i)=u(i)$ and $\left.\bar{p}_{x}\right|_{N-\{i\}}=\left.p_{x}\right|_{N-\{i\}}$. Because $f\left(p_{x}\right)=x$ and $(x, a) \in p_{x}(i)$, where $x \neq a$, it follows that $f$ is manipulable at $\bar{p}_{x}$ by agent $i$ towards profile $p_{x}$. This contradiction yields that $f(w)=x$ and $f$ is image-dictatorial with image-dictator $i$.

Finally, we consider the case where $U=\emptyset$. First we introduce an example which expresses a sufficient condition. Then like in the case that $U$ is a singleton we show that this sufficient condition is also necessary.

Example 3 Let $U=\emptyset$. Let $a, b \in A$, with $a \neq b$, such that $(a, b) \notin P^{1} \cup P^{2}$ 
and $(b, a) \notin P^{1} \cup P^{2}$. Define choice rule $f$ for a profile $p$ as follows

$$
\begin{aligned}
f(p) & =b \text { if both }(b, a) \in p(1) \text { and }(b, a) \in p(1) \\
& =a \text { in all other cases. }
\end{aligned}
$$

Like in Example 2 it follows that $f$ is unanimous, strategy-proof and non-imagedictatorial.

Lemma 4 Let $U=\emptyset$. hen the following two are equivalent

1. There are different agents $i$ and $j$ and alternative $a, b \in A$ such that $(a, b) \notin$ $P^{i} \cup P^{j}$ and $(b, a) \notin P^{i} \cup P^{j}$;

2. There exist unanimous, strategy-proof and non-image-dictatorial choice rules $f$ from $\mathbb{V}^{N}$ to $A$.

Proof. ((1) implies (2)) This implication follows by Example 3.

((2) implies (1)) To prove the contra position of this implication suppose

$(\alpha)$ For all alternatives $a, b \in A$, with $a \neq b$ there are agents $i_{a b}$ such that for all $j \in N-\left\{i_{a b}\right\}(a, b) \in P^{j}$ or $(b, a) \in P^{j}$

( $\beta$ ) Choice rules $f$ from $\mathbb{V}^{N}$ to $A$ is unanimous and strategy-proof.

It is sufficient to proof that $f$ is image-dictatorial. If $f\left(\mathbb{V}^{N}\right)$ is a singleton, then $f$ is a constant choice rule and all agents are image-dictator. So, let $f\left(\mathbb{V}^{N}\right)$ consist of more than one element. Because $\mathbb{V}^{N}$ is a convex restricted domain we may assume that there are an agent $i$, alternatives $a$ and $b$, with $a \neq b$, and profiles $p_{a}$ and $p_{b}$ such that $f\left(p_{a}\right)=a, f\left(p_{b}\right)=b,\left.p_{a}\right|_{N-\{i\}}=\left.p_{b}\right|_{N-\{i\}}$ and $p_{a}(i)=\left(p_{b}(i)-\{(b, a)\}\right) \cup\{(a, b)\}$. From assumption $(\alpha)$ we infer that $i=i_{a b}$. Next let $X_{a}=\left\{p \in \mathbb{V}^{N} \mid p(i)=p_{a}(i)\right\}$ and let $X_{b}=\left\{p \in \mathbb{V}^{N} \mid p(i)=p_{b}(i)\right\}$. We show that $f\left(X_{a}\right)=\{a\}$ and $f\left(X_{b}\right)=\{b\}$. Let $q_{a}$ and $q_{b}$ by $j$-deviations of $p_{a}$ and $p_{b}$ respectively from some agent $j \in N-\{i\}$, such that $\left.p_{a}\right|_{N-\{j\}}=$ $\left.q_{a}\right|_{N-\{j\}},\left.p_{b}\right|_{N-\{j\}}=\left.q_{b}\right|_{N-\{j\}}$ and $q_{a}(j)=q_{b}(j)=\left(p_{a}(j)-\{(x, y)\}\right) \cup\{(y, x)\}$ for some alternatives $x, y \in A$. Because $\mathbb{V}^{N}$ is convex restricted in order to prove $f\left(X_{a}\right)=\{a\}$ and $f\left(X_{b}\right)=\{b\}$ it is sufficient to prove that $f\left(q_{a}\right)=a$ and $f\left(q_{b}\right)=b$. Assumption $(\alpha)$ yields that $j=i_{x y}$. But as $j \neq i$ and $i=i_{a b}$, this implies by assumption $(\alpha)$ that $\{a, b\} \neq\{x, y\}$. If $a \notin\{x, y\}$, then by Maskin monotonicity we have $f\left(q_{a}\right)=a$ and $f\left(q_{b}\right) \neq a$. But as Strong Positive Association and $f\left(q_{a}\right)=a$ imply $f\left(q_{b}\right) \in\{a, b\}$ it follows that $f\left(q_{b}\right)=b$. Hence, if $a \notin\{x, y\}$, then $f\left(q_{a}\right)=a$ and $f\left(q_{b}\right)=b$. Similarly it follows that if $b \notin\{x, y\}$, then $f\left(q_{a}\right)=a$ and $f\left(q_{b}\right)=b$. Because $\{a, b\} \neq\{x, y\}$, at least one of these implications hold and therewith $f\left(q_{a}\right)=a$ and $f\left(q_{b}\right)=b$. Hence, $f\left(X_{a}\right)=\{a\}$ and $f\left(X_{b}\right)=\{b\}$.

For $R \in \mathbb{V}^{i}$ let $X_{R}=\left\{p \in \mathbb{V}^{N} \mid p(i)=R\right\}$. Hence, $X_{a}=X_{p_{a}(i)}$ and $X_{b}=X_{p_{b}(i)}$. The following two claims complete the proof.

Claim 1 Let $R \in \mathbb{V}^{i}$ be such that $f\left(X_{R}\right)=\{x\}$. Then $x=\operatorname{best}\left(\left.R\right|_{f\left(\mathbb{V}^{N}\right)}\right)$. 
To the contrary let $x \neq y=\operatorname{best}\left(\left.R\right|_{f\left(\mathbb{V}^{N}\right)}\right)$. Then there are $p_{y} \in \mathbb{V}^{N}$ such that $f\left(p_{y}\right)=y$. Consider $i$-deviation $p_{x y}$ of $p_{y}$ such that $\left.p_{x y}\right|_{N-\{i\}}=\left.p_{y}\right|_{N-\{i\}}$ and $p_{x y}(i)=R$. Because $f\left(X_{R}\right)=\{x\}$ we have $f\left(p_{x y}\right)=x$ and herewith $f$ is manipulable by agent $i$ at $p_{x y}$ towards profile $p_{y}$.

Claim 2 Let $R^{1}$ and $R^{2}$ be two relations in $\mathbb{V}^{i}$ that form an elementary change in $x, y \in A$, i.e. $R^{1}=\left(R^{2}-\{(y, x)\}\right) \cup\{(x, y)\}$. Let $f\left(X_{R^{1}}\right)=\{c\}$ for some alternative $c$ in $A$. Then $f\left(X_{R^{2}}\right)=\{d\}$ for some alternative $d$ in $A$.

In case $x \neq c$ Maskin monotonicity and $f\left(X_{R^{1}}\right)=\{c\}$ imply $f\left(X_{R^{2}}\right)=\{c\}$. So, suppose $c=x$. We are also done if $f\left(X_{R^{2}}\right)=\{c\}$. As $\mathbb{V}^{i}$ is a convex restricted domain we therefore may assume that there are $p_{c} \in X_{R^{1}}$ and $p_{d} \in$ $X_{R^{2}}$ where $c=f\left(p_{c}\right) \neq f\left(p_{d}\right)=d$ and $\left.p_{c}\right|_{N-\{i\}}=\left.p_{d}\right|_{N-\{i\}}$. Now similarly to the proof of $f\left(X_{a}\right)=a$ and $f\left(X_{b}\right)=b$ it follows that $f\left(X_{R^{2}}\right)=\{d\}$.

Claims 1 and 2 and the fact that $\mathbb{V}^{N}$ is a convex restricted domain imply that $f$ is image-dictatotrial.

Summarizing the above results yields

Corollary 1 Let $\mathbb{V}^{N}$ be a convex restricted domain. Hence, for all agents $i$ $\mathbb{V}^{i}=\left\{R \in \mathbb{L}(A) \mid P^{i} \subseteq R\right\}$ for some partial ordering $P^{i}$ referred to as $i$ 's a priori information. Let $P^{*}=\cup\left\{P^{i} \mid i \in N\right\}$ denote the collective a priori information. Let $U=\operatorname{undom}\left(P^{*}\right)$ be the set of undominated elements with respect to this collective a priori information $P^{*}$. Then (1) and (2) are equivalent, where

1. There exist non-image-dictatorial, unanimous and strategy-proof choice rules $f$ from $\mathbb{V}^{N}$ to $A$;

2. Either one of the following three

a There are two alternatives $a$ and $b$ such that $U=\{a, b\}$;

b There is one alternative a such that $U=\{a\}$ and there is an alternative $b \in A-\{a\}$ and there are two different agents, say $i$ and $j$, such that $(a, b) \notin P^{i} \cup P^{j}$;

c Set $U$ is empty and there are two different alternatives, say a and $b$, and two different agents, say $i$ and $j$, such that $(a, b) \notin P^{i} \cup P^{j}$ and $(b, a) \notin P^{i} \cup P^{j}$.

\section{Conclusions}

Corollary 1 describes the convex restricted domains which allow for unanimous, non-image-dictatorial and strategy-proof choice rules. The main requirement that there are at most two undominated alternatives with respect to the united a priori information is considerably restrictive. Therefore the characterization can also be seen as an impossibility result. On the other hand these conditions would only change marginally if anonymity were taken instead of non-imagedictatorship. Where anonymity is a condition that imposes that the choice rule 
can be defined on the frequency distributions of the preferences in a profile. So, agents are treated equally. Indeed considering Example 1 it is easy to define anonymous choice rules on two alternatives. The case of two undominated alternative would not change at all. In case of one or no undominated alternative differences occur because for instance a constant rule is anonymous but also image-dictatorial.

Another condition frequently imposed on choice rules is Pareto-optimality. It means that an alternative $x$ is not chosen when there are alternatives $y$ which all agents (strictly) prefer to $x$. It is not difficult to see that the choice rules of Example 1 are not necessarily Pareto-optimal. There are however special cases of this example which allow for Pareto-optimal, non-image dictatorial and strategy-proof choice rules. To illustrate this take the same notations and assumptions as in Example 1. Furthermore, assume that for all $c \in A-\{a, b\}$

$(a, c) \in P^{*}$. Now the following imputation rule $f$ is well-defined and satisfies these three conditions.

$$
\begin{aligned}
f(p) & =b \text { if }(b, a) \in p(i) \text { for all agents } i \in N \\
& =a \text { in all other cases. }
\end{aligned}
$$

Pareto-optimality is a stronger condition than unanimity. In view of the restrictive result spelled out by Corollary 1 we therefore did not incorporate an extensive study on convex restricted domains which allow for Pareto-optimal, non-(image)-dictatorial and strategy-proof choice rules.

Based on Corollary 1 and the remarks made above one might argue that changing the conditions of admissible choice rules will not lead to more general convex restricted domains which allow for such rules. Hence the concept of convexity is too restrictive after all. Indeed if we weaken the convexity condition, i.e. that all intermediate preferences of two admissible preferences are admissible, into connectedness, i.e. there is a path of intermediate preferences leading from one to the other admissible preference, then more general restricted domains may allow for "nice " choice rules. For example the domains discussed here as well those of single peaked preferences fall under this connectedness condition and both allow for acceptable choice rules.

\section{References}

1. Arrow K.J.,1978, Social choice and individual values, Yale University Press (19 ${ }^{\text {th }}$ edition).

2. Black D., 1948, On the rationale of group decision making, Journal of Political Economy,56, p. 23-34.

3. Bochet O. and Storcken T., 2005, Maximal domains for strategy-proof or Maskin monotone choice rules, METEOR research memorandum, Maastricht.

4. Gaertner W., 2002, Restricted domains, in: K.J. Arrow, A.K. Sen and K. Suzumura (ed.), Handbook of Social Choice, 1, chapter 3, p. 131-170, Elsevier.

5. Gibbard A., 1973, Manipulation of voting schemes: A general result, Econometrica, 41, p. 587-601. 
6. Inada K., 1964, A note on the simple majority decision rule, Econometrica, 32, p. 525-531.

7. Kalai E. and Muller E., 1977. "Characterization of domains admitting nondictatorial social welfare

functions and nonmanipulable voting procedures." Journal of Economic Theory, 16, p.457 469 .

8. Kalai E. and Ritz Z., 1980. "Characterization of the private alternatives domains admitting Arrow

social welfare functions." Journal of Economic Theory, 22, p.23 36.

9. Kemeny J.G. and Snell J.L., 1962, Mathematical models in social sciences, New York, Ginn and Company.

10. Moulin H., 1980, On strategy-proofness and single peakedness, Public Choice, 35, p.437-455.

11. Ritz. Z., 1985, Restricted domains, Arrow social welfare functions and noncorruptable and nonmanipulable social choice correspondences: The case of private alternatives, Mathematical Social Science, 4, p.155-179.

12. Satterthwaite M.A., 1975, Strategy-proofness and Arrow's conditions: Existence and correspondence theorem for voting procedures and social welfare functions, Journal of Economic Theory, 10, p.187-217.

13. Sen A.K. and Pattanaik P.K., 1969, Necessary and sufficient conditions for rational choice under majority decision, Journal of Economic Theory, 1, p.178-202.

14. Storcken T., 1985, Societies with a priori information and anonymous social welfare functions, Methods of Operations Research, 54, p. 279-291. 